\title{
Pulsar Kicks With Sterile Neutrinos and Landau Levels
}

\author{
Leonard S. Kisslinger \\ Department of Physics, Carnegie Mellon University, Pittsburgh, PA 15213 \\ Ernest M. Henley \\ Department of Physics, University of Washington, Seattle, WA 98195 \\ Mikkel B. Johnson \\ Los Alamos National Laboratory, Los Alamos, NM 87545
}

\begin{abstract}
We use a model with two sterile neutrinos obtained by fits to the MiniBoone and LSND experiments. Using formulations with neutrinos created by URCA processes in a strong magnetic field, so the lowest Landau level has a sizable probability, we find that with known paramenters the asymmetric sterile neutrino emissivity might account for large pulsar kicks.
\end{abstract}

PACS Indices:97.60.Bw,97.60.Gb,97.60.JD

\section{INTRODUCTION}

The gravitational collapse of a massive star often leads to the formation of a neutron star, a pulsar. It has been observed that many pulsars move with linear velocities of $1000 \mathrm{~km} / \mathrm{s}$ or greater. See Ref. [1] for a review. We have investigated the pulsar kicks which arise from the modified URCA processes in the time interval 10-20 sec after the supernova collapses, with a strong magnetic field and temperature so that the population of the lowest Landau level is approximately 0.4 of the total occupation, and we find large pulsar kicks [2].

The largest neutrino emission after the supernova collapse takes place during the first 10 seconds, with URCA processes dominant. The possibility of pulsar kicks from anisotropic neutrino emission due to strong magnetic fields during this time was discussed more than two decades ago 3 . It has been shown[4] that, with the strength of the magnetic field expected during this period, the lowest Landau level has a sizable occupation probability, which produces the neutrino emission asymmetry that is needed for pulsar kicks. However, due to the high opacity for standard model neutrinos in the dense region within the neutrinosphere, few neutrinos are emitted, and the pulsar kick is not obtained[5]. Sterile neutrinos with a small mixing angle have small opacities. It has been shown [6] that using the model of Ref.[4] and assuming the existence of a heavy sterile neutrino (mass $>1 \mathrm{kev}$ ), with mass and mixing angle constrained to fit dark matter, the pulsar kicks might be explained.

Recently, the MiniBooNE Collaboration found that the data for electron neutrino appearance showed an excess at low energies, in comparison to what was expected in the standard model [7]. This data, along with the LSND data, has been analyzed in a model with two light sterile neutrinos [8], and compared to MiniBooNE data [9]. The mixing angles of two light sterile neutrinos were extracted. See, however, Ref. [10], which questions the accuracy of the results of $\operatorname{Ref}[8]$.

In the present paper we use the fits of Refs. [8, 9] with two sterile neutrinos to investigate the possibility of obtaining the large pulsar velocities which have been observed. As we shall show, our model differs from that of Ref [6] in that with a much larger mixing angle there is a higher probability of sterile neutrinos, but a much smaller effective volume, due to a larger opacity. However, as we shall show, since the mean free path is much larger than those of standard neutrinos, under the conditions in which standard neutrinos produce a pulsar velocity of 2$300 \mathrm{~km} / \mathrm{s}$, the MiniBoone/LSND sterile neutrinos can give a kick of more than $1000 \mathrm{~km} / \mathrm{s}$.

\section{ASYMMETRIC STERILE NEUTRINO EMISSIVITY AND PULSAR KICKS IN LIGHT TWO-STERILE NEUTRINO MODEL}

Within about 1 second after the gravitational collapse of a large star, the neutrinosphere with a radius of about $40 \mathrm{~km}$, with temperature equilibrium, is formed. For about 10 seconds about $98 \%$ of neutrino emission occurs, with neutrinos produced mainly by URCA processes. Due to the strong magnetic field, neutrino momentum asymmetry is produced within the neutrinosphere, but with a small mean free path they are emitted only from a small surface layer of the neutrinosphere, and the pulsar kick cannot be accounted for. If a standard active neutrino, say the electron neutrino, oscillates into a sterile neutrino, 
it will escape from the protoneutrino star and neutrinosphere, unless it oscillates back into the active neutrino. The mixing angle plays a key role. In the work of Fuller et al[6] the mixing angle is so small that the sterile neutrinos are emitted. In the present work the starting point is the analysis of MiniBooNE and LSND data, with the two or more sterile neutrinos with small masses and large mixing angles. Before we can proceed, however, it is essential to determine possible effects of the high density and temperature of the medium on the mixing angles.

\section{A. Mixing Angle in Neutrinosphere Matter}

It has long been known that dense matter can effect neutrino states. The famous MSW effect 11, 12] for understanding solar neutrinos, and the study of oscillations of high energy neutrinos 13] are studies of mixing of active neutrinos in matter. There have been many other studies. In the present work we are dealing with sterile/active neutrino mixing given by the mixing angle $\theta_{m}$ in neutrinosphere matter

$$
\begin{aligned}
& \left|\nu_{1}>=\cos \theta_{m}\right| \nu_{e}>-\sin \theta_{m} \mid \nu_{s}> \\
& \left|\nu_{2}>=\sin \theta_{m}\right| \nu_{e}>+\cos \theta_{m} \mid \nu_{s}>.
\end{aligned}
$$

In the work Ref[ $[\underline{6}]$ it was shown that the mixing angle for sterile neutrinos that can account for dark matter as well as those produced in the neutron star core is almost the same as the vacuum value. Starting from the much larger mixing angles for the sterile neutrinos that seem to account for the MiniBoonE, LSND data, we need the value of the mixing angles in the neutrinosphere, as we discuss below. The effective mixing angle in matter, $\theta_{m}$ can be related to the vacuum mixing angle, $\theta$ by [14]

$$
\sin ^{2}\left(2 \theta_{m}\right)=\frac{\sin ^{2}(2 \theta)}{\sin ^{2}(2 \theta)+\left(\cos (2 \theta)-\frac{2 p V^{T}}{(\delta m)^{2}}\right)^{2}} .
$$

In $\mathrm{Eq}(2) V^{T}$ is the finite temperature potential, while the finite density potential due to asymmetries in weakly interacting particles has been dropped as it vanishes when temperature equilibrium is reached [14]. A convenient form for $V^{T}$, with the background of both neutrinos and electrons included, is given in $\operatorname{Ref}[15]$

$$
V^{T}=\frac{28 \pi G_{F}^{2}}{45 \alpha} \sin ^{2} \theta_{W}\left(1+0.5 \cos ^{2} \theta_{W}\right) p T^{4},
$$

with $G_{F}, \theta_{W}$ the standard weak interaction parameters and $\alpha=1 / 137$. Assuming $\mathrm{T}=20 a \mathrm{MeV}$, with $a \leq 1.0, \mathrm{p}=b \mathrm{MeV}$, and $(\delta m)^{2}=1.0 \mathrm{ev}^{2}[8,[10]$, we find

$$
\frac{2 p V^{T}}{(\delta m)^{2}} \simeq 5.1 \times 10^{-3} b^{2} a^{4} \ll \cos (2 \theta) .
$$

Therefore the mixing angle in the neutrinosphere medium is approximately the same the vacuum mixing angle. This agrees with $\operatorname{Ref}[6]$.

\section{B. Emissivity With a Light Sterile Neutrino}

We now use the fits to MiniBooNe and LSND with light sterile neutrinos to estimate pulsar kicks. The MiniBooNE results are consistent with the LSND results only if there are at least two sterile neutrinos. Models with three sterile neutrinos have also been considered [10, 16]. Fits to the MiniBooNE experiment and the LSND results by Ref. [8] in Ref.[9] with two sterile neutrinos are shown in Fig. 1. M. Maltoni and T. Schwetz, hep-ph/0705.0107 (2007)

Figure by M. Sterbenz, LANL

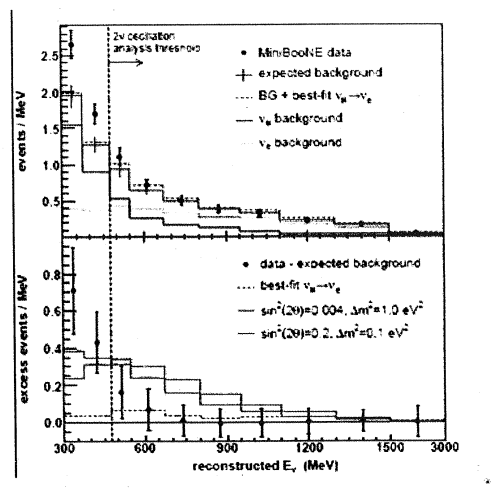

FIG. 1: $\sin ^{2}\left(2 \theta_{1 s}\right)=0.004 ; \sin ^{2}\left(2 \theta_{2 s}\right)=0.2$ 
From the Sterbenz/Maltoni-Schwetz fits one finds for the mixing angles of the two sterile neutrinos:

$$
\begin{aligned}
& \left(\sin 2 \theta_{1 s}\right)^{2}=0.004 \\
& \left(\sin 2 \theta_{2 s}\right)^{2}=0.2,
\end{aligned}
$$

and the masses are negligibly small. Note that this is in contrast to the parameters of Ref.[ [6] , with the constraint of dark matter giving a mixing angle of $\left(\sin 2 \theta_{d m}\right)^{2} \simeq 10^{-8}$, and a mass greater than $1 \mathrm{keV}$. Note that in a recent analysis by Maltoni [10] it was found that the fit [8] to the MiniBooNE and LSND data had problems with disappearance data, although there are rather large error bars. In our present work we will use values for $(\sin 2 \theta)^{2}$ in the range 0.2 to 0.004 to estimate the pulsar kick.

The probability of asymmetric emission, giving a pulsar kick, does not depend directly on the sterile neutrino mass in our model, but is proportional to the $\left(\sin 2 \theta_{s}\right)^{2}$. It is the large mixing angles found in fits to MiniBoone and LSND that lead us to carry out the investigation in the present paper.

\section{General Formulation of Neutrino Emissivity With a Strong Magetic Field}

The neutrino emissivity is given in general form in many papers, e.g., see Refs [17, 18]:

$$
\begin{aligned}
e^{\nu}= & \Pi_{i=1}^{4} \int \frac{d^{3} p^{i}}{(2 \pi)^{3}} \frac{d^{3} q^{\nu}}{2 \omega^{\nu}(2 \pi)^{3}} \int \frac{d^{3} q^{e}}{(2 \pi)^{3}} \\
& (2 \pi)^{4} \sum_{s_{i}, s^{\nu}} \frac{1}{2 \omega_{L}^{e}} \omega^{\nu} \mathcal{F} M^{\dagger} M \\
& \delta\left(E_{\text {final }}-E_{\text {initial }}\right) \delta\left(\vec{p}_{\text {final }}-\vec{p}_{\text {initial }}\right),
\end{aligned}
$$

where $M$ is the matrix element for the URCA process and $\mathcal{F}$ is the product of the initial and final Fermi-Dirac functions corresponding to the temperature and density of the medium. The main source of the asymetric emissivity that produces the pulsar velocity is the fact that the electron has a large probability to be in the lowest $(\mathrm{n}=0)$ Landau level. See Refs [19, 20] for a discussion of Landau levels. The asymetric emissivity can be seen by considering the weak axial intereaction, $W_{A}$,

$$
\begin{aligned}
W_{A} & =-\frac{G}{\sqrt{2}} g_{A} \chi_{p}^{\dagger} \vec{l} \cdot \vec{\sigma} \chi_{n} \\
l_{\mu} & =\bar{\Psi}\left(q^{e}\right) \gamma_{\mu}\left(1-\gamma_{5}\right) \Psi\left(q^{\nu}\right),
\end{aligned}
$$

with $G=\frac{10^{-5}}{m_{n}^{2}}, g_{A}=1.26$, the $\chi$ are the nucleon spinors, and the lepton wave functions are
$\Psi\left(q^{e}\right), \Psi\left(q^{\nu}\right)$, where $q^{e}$ and $q^{\nu}$ are the electron and antineutrino momenta, respectively. The key to the asymmetric emission is given be the trace over the leptonic currents, $\operatorname{Tr}\left[l_{i}^{\dagger} l_{j}\right]$,

$$
\begin{aligned}
\int d^{2} q_{\perp}^{e} \operatorname{Tr}\left[l_{i}^{\dagger} l_{j}\right] \simeq & 8 \pi E^{e}\left[\left(q^{\nu}\right)^{j} \delta_{i 3}+\left(q^{\nu}\right)^{i} \delta_{j 3}\right. \\
& \left.-\delta_{i j}\left(q^{\nu}\right)^{3}\right]\left(\hat{q}^{e}=\hat{B}=\hat{z}\right),
\end{aligned}
$$

with the magnetic field $\mathrm{B}$ in the $\mathrm{z}$ direction. We only consider the weak axial force, which is dominant. Using the relationship given in $\mathrm{Eq}(8)$ one can show that the result of the traces and integrals over the axial product matrix element has the form $(\hat{B}=\hat{z})$

$$
\iint\left|M_{A}\right|^{2} \propto\left(q^{\nu}\right)^{z} .
$$

Details are given in Ref [2], where it is shown that the asymmetric neutrino emissivity, using the general formulation of Ref [17], is

$$
\begin{aligned}
\epsilon^{A S} \simeq & 0.64 \times 10^{21} T_{9}^{7} P(0) \times f \\
& \quad \operatorname{erg} \mathrm{cm}^{-3} \mathrm{~s}^{-1}=p_{n s} c \frac{1}{V_{e f f} \Delta t},
\end{aligned}
$$

where $T_{9}=T /\left(10^{9} \mathrm{~K}\right), p_{n s}$ is the neutron star momentum, $P(0)$ is the probability of the electron produced with the antineutrino being in the lowest Landau state, $\mathrm{f}=.52$ is the probability of the neutrino being at the $+\mathrm{z}$ neutrinosphere surface [2], $V_{\text {eff }}$ is the volume at the surface of the neutrinosphere from which neutrinos are emitted, and $\Delta t \simeq 10 \mathrm{~s}$ is the time interval for the emission.

We derive $P(0)$ and $V_{\text {eff }}$, the effective volume for the emissivity, in the next two subsections.

\section{D. $P(0)=$ Probability for the Electron to be in the $n=0$ Landau Level}

Just as in our previous work in which Landau levels play a crucial role 2], only the lowest Landau level, for which the helicity is $-1 / 2$ (rather than $\pm 1 / 2$ as with the usual Dirac spinors) gives asymmetric emission. The probability that an electron in a strong magnetic field is in the lowest $(\mathrm{n}=0)$ Landau level, $P(0)$, can be calculated from the temperature, $\mathrm{T}$, and the energy spectrum of Landau levels [19, 20]. A particle with momentum $\mathrm{p}$ and effective mass $m_{e}^{*}$ in a magnetic field B in the nth Landau level has the energy

$$
E^{L}(p, n)=\sqrt{p^{2}+\left(m_{e}^{*}\right)^{2}+2\left(m_{e}^{*}\right)^{2} \frac{B}{B_{c}} n},
$$


with $B_{c}=4 \times 10^{13} \mathrm{G}$, and $m_{e}^{*}$ is the effective mass of the electron at the high density of the protoneutron star and neutrinosphere.

From standard thermodynamics the probability of occupation of the $\mathrm{n}=0$ Landau level, $\mathrm{P}(0)$, is given by [] $]$ :

$$
P(0)=\frac{F(0)}{F(0)+2 \sum_{1}^{\infty} F(n)},
$$

where $\mathrm{F}(\mathrm{n})$, with magnetic field $\mathrm{B}$, temperature $\mathrm{T}$, and chemical potential $\mu$, is

$$
F(n)=\int_{p_{\min }}^{\infty} d p \frac{\left[m_{n}-m_{p}-E^{L}(p, n)\right]^{2}}{1+\exp \left[\left(E^{L}(p, n)-\mu\right) / T\right]}(1
$$

The electron energy is restricted to magnitudes greater than $\mu$, but the integrals in Eq.(77) are insensitive to $p_{\text {min }}$, so we take $p_{\min }=0$ as in $\operatorname{Ref}[6]$.

We agree with the estimate of Ref.[6] for $\mathrm{P}(0)$. Note that if we had used the free electron mass, $m_{e}$, in the Landau energies (Eq. (5) ) we would have obtained a much smaller value for $\mathrm{P}(0)$. For $\mathrm{B}=10^{16}$ $\mathrm{G}, \mu=40 \mathrm{MeV}, m_{e}^{*}=4 \mathrm{MeV}$ and $T_{\nu \text {-sphere }}=20$ $\mathrm{MeV}, \mathrm{P}(0) \simeq 0.3$. This is similar to our estimate of $\mathrm{P}(\mathrm{n}=0) \simeq 0.4$ at the surface of the protoneutron star at about 10 seconds [2]. Therefore our result for asymmetric emissivity differs from that of Fuller et al [6] mainly in that we have a much larger mixing angle, and a much smaller effective volume, since the sterile neutrinos oscillate back to active neutrinos within the neutrinosphere; and therefore our emission only takes place near the surface of the neutrinoshere. However, in contrast with purely active neutrino emission in which the opacity results in very small pulsar kicks [5], the sterile neutrinos have a much larger effective volume, and can therefore produce much larger pulsar velocities

\section{E. Estimate of $V_{e f f}=$ Effective Volume for Emission}

To estimate $V_{\text {eff }}$ we make use of the early study of opacity in about the first 20 s of the creation of a neutron star via a supernova collapse [21, 23, 24], and a recent detailed study of neutrino mean free paths 22]. Since the mean free path of the sterile neutrino is determined by that of the standard neutrino to which it oscillates, $\lambda$, we make use of studies of active neutrino mean free paths. First note that the neutrino mean free path is given by

$$
1 / \lambda=\int \frac{d^{3} p}{(2 \pi)^{3}} M_{f i}[1-n(q)]
$$

$$
\times\left(1+e^{\left(\mu_{\nu}-E_{\nu}\right) / k T}\right),
$$

where $M_{f i}$ is the weak matrix element and $n(q)$ is the Fermi distribution. For the calculation of the sterile neutrino, for which $M_{f i}=0$, one can use the value of $1 / \lambda$ with a factor of $\sin ^{2}(2 \theta)$ from the matrix element and another such factor from the occupation probability. From the results of previous authors, for $\mathrm{T}$ in the 10 to $20 \mathrm{Mev}$ range and $\mu$ in the 20 to $40 \mathrm{MeV}$ range, we estimate that $\lambda \simeq 1.0 \mathrm{~cm}$ This gives a range for the effective sterile neutrino mean free path

$$
\lambda_{s} \simeq 5.0 \text { to } 250 \mathrm{~cm} .
$$

For a neutrinosphere radius of $40 \mathrm{~km}$, with $\lambda_{s}<<$ $R_{\nu}$ this gives us $V_{\text {eff }}=(4 \pi / 3)\left(R_{\nu}^{3}-\left(R_{\nu}-\lambda_{s}\right)^{3}\right) \simeq$ $4 \pi R_{\nu}^{2} \lambda_{s}$.

From Eq.(10), $R_{\nu}=40 \mathrm{~km}$, and $\lambda=1.0 \mathrm{~cm}$,

$$
p_{n s}=M_{n s} v_{n s} \simeq \frac{0.67 \times 10^{25}}{\sin ^{2}(2 \theta)} T_{9}^{7} \mathrm{gm} \frac{\mathrm{cm}}{\mathrm{s}},
$$

with $T_{9}=\frac{T}{10^{9} \mathrm{~K}}$. Taking the mass of the neutron star to equal the mass of our sun, $M_{n s}=2 \times 10^{33}$ $\mathrm{gm}$, we obtain for the velocity of the neutron star

$$
v_{n s} \simeq 3.35 \times 10^{-7}\left(\frac{T}{10^{10} K}\right)^{7} \frac{1}{\sin ^{2}(2 \theta)} \frac{k m}{s},
$$

For example, for $\mathrm{T}=10 \mathrm{Mev}=1.16 \times 10^{11} \mathrm{kK}$,

$$
v_{n s} \simeq 47.3 \frac{\mathrm{km}}{\mathrm{s}} \text { to } 2,370 \frac{\mathrm{km}}{\mathrm{s}},
$$

which means that sterile neutrino emission could account for the large pulsar kick with the parameters extracted from Refs [8, 9]. If we use the physical parameters that give Eq.(18) for electron neutrinos, we obtain a pulsar velocity of $v_{n s}=95 \mathrm{~km} / \mathrm{s}$, which is consistent with previous predictions by several authors.

It should be noted that the study of the MiniBooNE and LSND results are in progress, and the mixing angles that result could be much different from those which we have used, changing our results. Preliminary data from the MiniBooNE/Minos experiment 25 is consistent with the MiniBooNE results [7]. We also once more point out that Ref [10] questions the accuracy of the peramaters extracted by Ref [8]. 


\section{CONCLUSIONS}

Because of the strong magnetic fields in protoneutron stars and the associated neutrinosphere, the electrons produced in the URCA processes that dominate neutrino production in the first 10 seconds have a sizable probability, $\mathrm{P}(0)$, to be in the lowest $(n=0)$ Landau level. This leads to asymmetric neutrino momentum. With the mixing angles found in Refs [8, 9], we find that the sterile neutrinos produced during this period for high luminosity pulsars can give the pulsars velocities of greater than $1000 \mathrm{~km} / \mathrm{s}$, as observed, similar to predictions based on sterile neutrinos as dark matter 6$]$. Although the parameters found in the analyis [8, 9] of the MiniBooNE 7] and LSND data is still in question, current analysis of the FermiLab experiment 25] seems to be in agreement with MiniBooNE[7].

There is a strong correlation of the pulsar velocity with temperature, T. Since it is difficult to determine $\mathrm{T}$ accurately, it is difficult for us to predict the ve- locity of a pulsar whose kick arises from sterile neutrino emission. On the other hand, if the pulsar kick arises from the asymmetric emission of active neutrinos produced by the modified URCA processes after 10 seconds, also proportional to $\mathrm{P}(0)$ 2], then $\mathrm{T}$ can be determined by an accurate measurement of the neutrinos from the supernova. Therefore, in future years, with much more accurate neutrino detectors, one could predict the velocity of the resulting pulsar. Unfortunately, the energy of emitted sterile neutrinos cannot be measured. From our results in the present paper and those in Ref[2], high luminosity pulsars receive a large kick both from sterile neutrinos in the first ten seconds and standard neutrinos in the second ten seconds.

This work was supported in part by DOE contracts W-7405-ENG-36 and DE-FG02-97ER41014. The authors thank Terry Goldman; and William Louis, Gerald Garvey and other LANL members of the MiniBooNE Collaboration for helpful discussions.
[1] B.M.S. Hansen and E.S. Phinney, astro-ph/9708071, Mon. Not. R. Astron. Soc. 291, 569 (1997)

[2] E. M. Henley, M. B. Johnson and L. S. Kisslinger, astro-ph/0706.1511, Phys. Rev. D 76,125007 (2007)

[3] N.N Chugai, Sov. Astron. Lett. 10, 87 (1984)

[4] O.F. Dorofeev, V. N. Rodionov and I. M. Ternov, Sov. Astron. Lett. 11, 123 (1985)

[5] D. Lai and Y-Z Qian, ApJ. 505, 844 (1998). This article has references to earlier work on standard neutrinos and pulsar kicks.

[6] G. M. Fuller, A. Kusenko, I. Mocioiu and S. Pascoli, Phys. Rev. D 68, 103002 (2003)

[7] A.A. Aguilar-Arevalo et al (MiniBooNE Collaboration), Phys. Rev. Lett. 98, 231801 (2007)

[8] M. Maltoni and T. Schwetz, Phys. Rev. D76, 0930005 (2007)

[9] M. Sterbenz, LANL, MiniBooNE TN 225

[10] M. Maltoni, J. Phys. Conf. Ser. 110, 082011 (2008)

[11] L. Wolfenstein, Phys. Rev. D17, 2369 (1978); D20, 2634 (1979)

[12] S.P. Mikheyev and A. Yu Smirnov, Sov. J. Nucl. Phys. bf 42, 913 (1985)

[13] E.Kh. Akhmedov, M. Maltoni and A. Yu. Smirnov, Phys. Rev. Lett. 95, 211801 (2005)

[14] K. Abazajian, G.M. Fuller and M. Patel,Phys. Rev.
D64, 023501 (2001)

[15] D. Nötzold and G. Raffelt, Nulc. Phys. B307, 924 (1988)

[16] G.J. Stephenson, T. Goldman, B.H.J. McKellar and M. Garbutt, Int. J. Mod. Phys. A20, 6373 (2005); T. Goldman, G.J. Stephenson, Jr. and B.H.J. McKellar, Phys. Rev. D 75, 091301 (2007)

[17] B.L. Friman and O.V. Maxwell, ApJ 232, 541 (1979)

[18] J.N. Bahcall and R.A.Wolf, Phys. Rev. 140, B1452 (1965)

[19] M.H. Johnson and B.A. Lippman, Phys. Rev. 76, 828 (1949)

[20] J.J. Matese and R.F. O'Connel, Phys. Rev. 180, 1289 (1969)

[21] N. Iwanamoto and C.J. Pethik, Phys. Rev. D25, 313 (1982)

[22] I. Sagert and J. Schaffner-Bielich, J. Phys. G 35, 014062 (2008); astro-ph/0708.2352 (2007)

[23] A. Burrows and J.M. Lattimer, ApJ. 307, 178 (1986)

[24] A. Burrows, T.J. Mazurek and J.M. Lattimer, ApJ. 251, 325 (1981)

[25] R. Ospanov, Bulletin of APS April Meeting 2008, J2 3 